Case Report

\title{
Prostatic Abscess after Stapled Hemorrhoidopexy Caused by ESBL Extended Spectrum Beta Lactamase Producing Klebsiella pneumoniae: An Additional Challenge to Postoperative Sepsis
}

\author{
Asem Saleh, ${ }^{1}$ Abdelnasir Kibeida, ${ }^{2}$ Elsaid Amin, ${ }^{3}$ Abdalla Khalil, ${ }^{1}$ \\ Rafat Abu Shakra, ${ }^{4}$ and Mohamed Elwakil ${ }^{5}$ \\ ${ }^{1}$ International Medical Centre (IMC) Hospital, Internal Medicine Department, Jeddah, Saudi Arabia \\ ${ }^{2}$ IMC Hospital, General Surgery Department, Jeddah, Saudi Arabia \\ ${ }^{3}$ IMC Hospital, Radiology Department, Jeddah, Saudi Arabia \\ ${ }^{4}$ IMC Hospital, Clinical Pathology Department, Saudi Arabia \\ ${ }^{5}$ IMC Hospital, Emergency Medicine Department, Jeddah, Saudi Arabia
}

Correspondence should be addressed to Abdalla Khalil; abdallak59@gmail.com

Received 6 March 2017; Revised 2 June 2017; Accepted 19 June 2017; Published 17 July 2017

Academic Editor: Imran Hassan

Copyright (C) 2017 Asem Saleh et al. This is an open access article distributed under the Creative Commons Attribution License, which permits unrestricted use, distribution, and reproduction in any medium, provided the original work is properly cited.

\begin{abstract}
Postoperative septic complications of hemorrhoids surgical interventions are rare, but very serious with high mortality rate. Early diagnosis and prompt therapy are essential to save patient's life. There are a good number of articles and case reports about these septic complications. We are presenting a case report of a prostatic abscess caused by extended spectrum beta lactamase (ESBL) producing Klebsiella pneumoniae after hemorrhoidopexy. Our patient was a healthy middle aged Saudi male who has no significant medical history apart from morbid obesity and recurrent urinary tract infections. ESBL producing K. pneumoniae could be detected only after aspiration of the prostatic abscess, but proper antibiotic was introduced intravenously on admission before culture of aspirate of the abscess was available. Antibiotic was continued for 30 days and abscess resolved completely. In our electronic search, we could not find any case report of prostatic abscess after stapled hemorrhoidopexy caused by ESBL producing organism. This is an additional challenge for treating physicians as these organisms are sensitive only to one group of antibiotics (carbapenem group).
\end{abstract}

\section{Introduction}

Septic complications after treatment of hemorrhoids are extremely rare, but these can be devastating and have resulted in a number of deaths [1].

Retroperitoneal abscess and sepsis have been reported after SH stapled hemorrhoidopexy [2,3].

Perineal necrosis, abscess, and septic shock have been also reported after SH $[4,5]$.

Sepsis following hemorrhoids treatment has been reported as early as 12 hours after therapy [2].

It can take up to 7 days to manifest clinically and a rare case of severe perineal sepsis has been reported after 38 days following SH $[4,6]$.
Sepsis, septic shock, and liver abscess have been also reported with other modalities of treating hemorrhoids as sclerotherapy and rubber band ligation $[7,8]$.

A retrospective study showed that septic complications after hemorrhoidectomy represent only $0.1 \%$ of all operated on patients (2840 patients) [9].

Studies showed that $\mathrm{SH}$ has a significant higher rate of recurrence and postprocedural tenesmus [10].

\section{Case Presentation}

49-year-old Saudi male was operated on electively for secondand third-degree hemorrhoids after two ER visits with rectal blood spotting. His past medical and surgical history were 
unremarkable apart from morbid obesity and recurrent urinary tract infections that required intravenous therapy in other hospital.

Indications for surgery were recurrent bleeding with second- and third-degree hemorrhoids.

Surgery was done under spinal anesthesia and intravenous sedation.

No Foley's catheter was inserted. Patient was placed in lithotomy posture.

Examination under general anesthesia revealed secondand third-degree hemorrhoids.

Procedure started by placing the anal dilator and fixing it with $2 / 0$ silk interrupted sutures.

Purse string anoscope was then used to place the purse string sutures on rectal submucosal level using Prolene 2/0, placing 5-6 bites at $4 \mathrm{~cm}$ distance from the anal verge.

Stapler device $33 \mathrm{~mm}$ Ethicon proximate (fixed anvil) stapler was then introduced and purse string snugly tied; the Prolene suture ends were then retrieved through the device housing using the suture threader.

Device was closed by marking and then fired.

An evenly excised mucosa was then retrieved, width of which is about $1.5 \mathrm{~cm}$.

Hemostasis was well secured with interrupted vicryl 3/0 sutures.

No major complications were present during the procedure.

As the patient weight was $114 \mathrm{kgm}$, height $172 \mathrm{~cm}$, and BMI 38.5., the only difficulty encountered during surgery was in placing the anal dilator and fixing it in a correct position.

The patient was stable during the procedure with minimal blood loss and was discharged to the postanesthesia recovery room.

Five days after discharge, he came back to ER with fever, dysuria, frequency, and urgency for two days. His temperature was $38.2^{\circ} \mathrm{C}$ and the rest of exam was unremarkable. His complete blood count and renal function tests were normal. Urinalysis showed leucocytes +2 , WBC $40 / \mathrm{HPF}$, and negative nitrates.

He was treated as acute cystitis with ciprofloxacin $500 \mathrm{mg}$ twice daily orally.

He visited ER again after another two days with fever, rigors, abdominal pain, repeated vomiting, and generalized weakness. No rectal bleeding, pain, or discharge was present.

He denied any history of extramarital sexual relation or sexually transmitted disease before.

By examination, he looked sick and dehydrated. Temperature was $38.9^{\circ} \mathrm{C}$, BP $110 / 70$, pulse $120 /$ min regular, and oxygen saturation $97 \%$ at room air.

His abdominal exam showed tender suprapubic area and perineum. There was no discharge or bleeding from anal orifice. Cardiac and respiratory exam were unremarkable.

His $\mathrm{CBC}$ complete blood count and renal function tests were normal. Urinalysis showed leucocytes +3 , WBC $>100$ / HPF with negative nitrates.

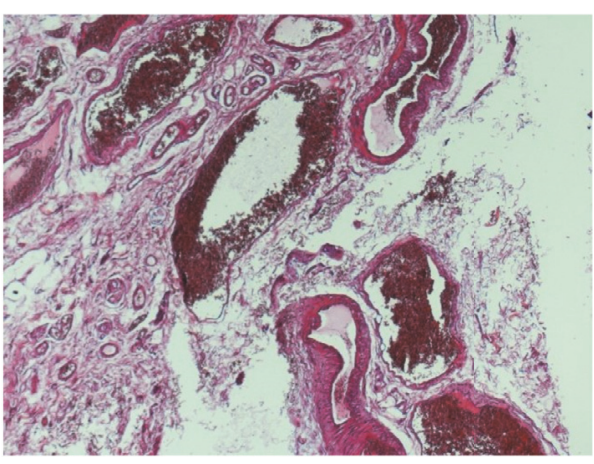

FIGURE 1: Photomicrograph shows hemorrhoids comprised of dilated, thick walled, congested submucosal blood vessels (hematoxylin and eosin stain, original magnification $\times 40$ ).

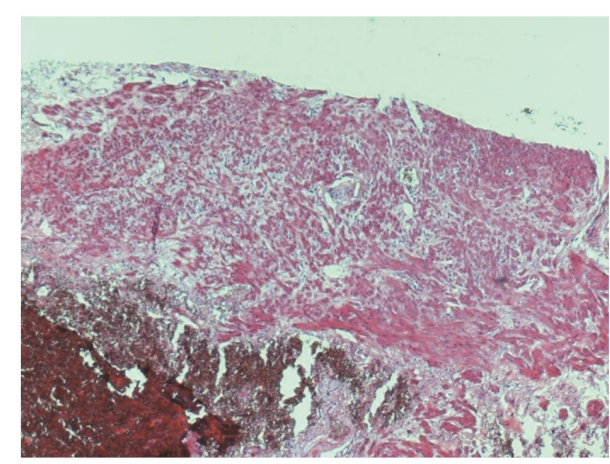

FIGURE 2: Photomicrograph shows portion of rectal muscularis propria identified at the deep aspect of the specimen (hematoxylin and eosin stain, original magnification $\times 40$ ).

Urine culture of previous ER visit was negative.

His CRP was raised $64 \mathrm{mg} / \mathrm{l}(0-5 \mathrm{mg} / \mathrm{l})$ and PSA total was $6 \mathrm{ug} / \mathrm{l}(0-4.4 \mathrm{ug} / \mathrm{l})$.

Both blood culture and urine cultures came to be negative. Both Chlamydia and gonococci antibody were negative.

Histopathology of the previous resected hemorrhoids specimen was reviewed.

It showed hemorrhoids comprised of dilated, thick walled, congested submucosal blood vessels (Figure 1). A portion of rectal muscularis propria was identified at the deep aspect of the specimen (Figure 2).

Abdomen and pelvis ultrasound at ER were unremarkable apart from enlarged prostate $45 \mathrm{cc}$. Patient was started empirically on meropenem intravenously 1 gm every 8 hours and he continued to be febrile for another 2 days.

On third day after admission, MRI of pelvis showed fluid intensity lesion within posterolateral aspect of the prostate gland measuring $24 \times 12 \times 14 \mathrm{~mm}$ with marginal enhancement (prostatic abscess) (Figure 3).

Infectious disease consultant and urologist suggested ultrasound guided transrectal aspiration of the abscess. 


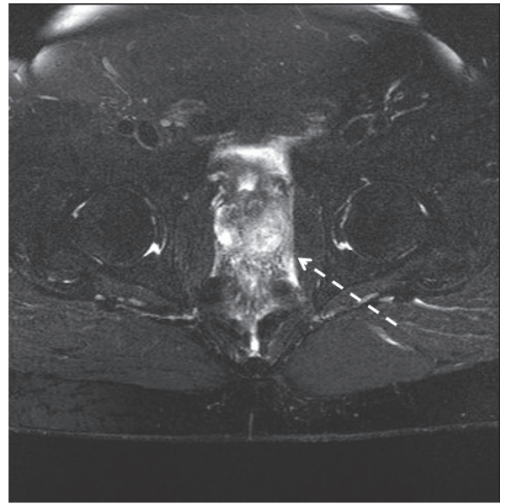

(a)

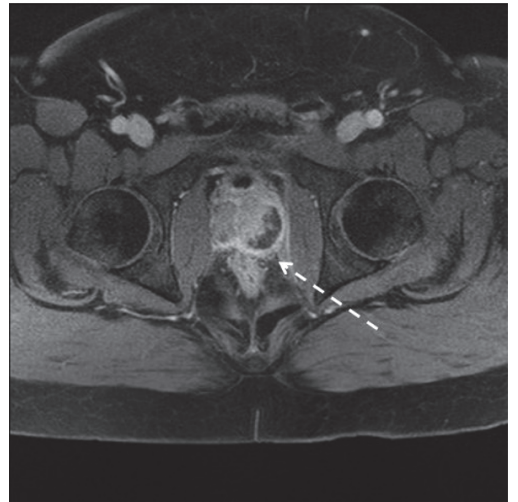

(b)

FIGURE 3: MRI of pelvis: axial FS T2WI (a), axial postcontrast T1WI (b). Before aspiration and antibiotic therapy showing fluid intensity lesion within posterolateral aspect of the gland measuring $24 \times 12 \times 14 \mathrm{~mm}$ with marginal enhancement (prostatic abscess).

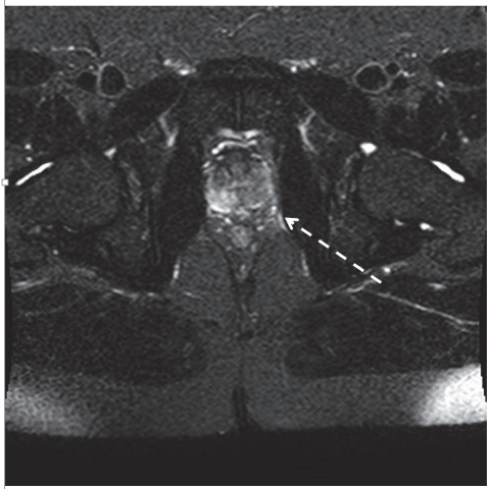

(a)

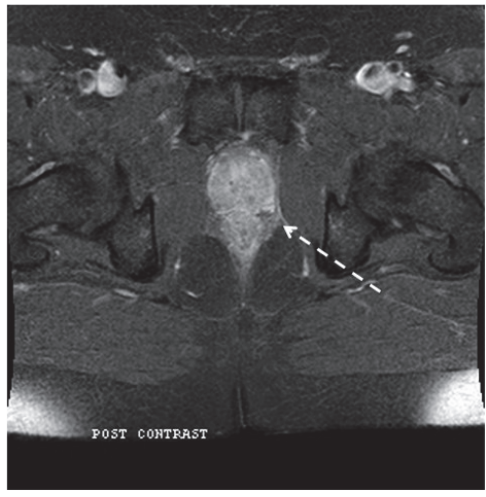

(b)

FIGURE 4: MRI of pelvis: axial FS T2WI (a), axial postcontrast T1WI (b). After aspiration and antibiotic therapy showing resolution of the previous intraprostatic collection.

Purulent fluid of $5 \mathrm{ml}$ was aspirated and sent for microbiology. It grew gram negative bacilli Klebsiella pneumoniae (morphology was done according to CDC algorithm). Klebsiella pneumoniae was resistant to cefuroxime, ceftazidime, ceftriaxone, and piperacillin/tazobactam and sensitive to ciprofloxacin, meropenem, imipenem, and gentamicin, that is, extended spectrum beta lactamase (ESBL) producing (culture sensitivity was done; MIC results obtained using automated Vitek 2 AST-GN69 and AST -XN06 cards).

Fever subsided next day after aspiration of abscess and patient felt well.

Meropenem continued for total of 14 days and then changed to ciprofloxacin $500 \mathrm{mg}$ bid and clindamycin $300 \mathrm{mg}$ q8 hours orally for total of 30 days. He was followed at infectious diseases clinic and repeated CRP was $6 \mathrm{mg}(0-5 \mathrm{mg} / \mathrm{l})$ and MRI showed resolution of the previous intraprostatic collection with residual edema along left perianal area due to previous surgery (Figure 4).

\section{Discussion}

Sepsis caused by MDR multidrug resistant bacteria represents an additional challenge to postoperative septic complications of hemorrhoids treatment.

Detection of these MDR bacteria will take 72 hours till we have culture sensitivity of aspirated fluid or blood culture and this will be reflected on outcome of the septic patient.

Extended spectrum beta lactamase (ESBL) producing Enterobacteriaceae (e.g., Escherichia coli and Klebsiella pneumoniae) is one of the challenging hospital acquired infections worldwide [11].

ESBL producing Escherichia coli (E. coli) and Klebsiella pneumoniae infections carry a higher mortality rate, higher risk of developing bacteremia, and failure of therapy compared to nonproducing ESBL isolates [12, 13].

Carbapenem group of antibiotics is the drug of choice for treating ESBL E. coli and Klebsiella pneumoniae infections. 
Prostatic abscess after SH in our case report could be explained by the possibility that purse string might have been a bit too profound, on the anterior side, taking not only mucosa and submucosa, but also a bite of rectal smooth muscle (Figures 1 and 2).

In our electronic search, we could not find any reported prostatic abscess caused by ESBL E. coli or Klebsiella pneumoniae infection as postoperative septic complications for hemorrhoids surgical intervention.

Our patient presented with fever and symptoms of genitourinary tract infection five days after hemorrhoidopexy. His urinalysis was nitrates negative and WBCs were 40/HPF, but urine culture was negative.

Treating team considered possibility of ESBL with patient's past history of recurrent urinary tract infections and intravenous therapy at other hospital.

Patient was started on meropenem empirically. Both blood culture and urine culture on admission came to be negative while purulent fluid aspirated from prostatic abscess grew ESBL K. pneumoniae.

With aspiration of the prostatic abscess, causative organism could be detected and resolution of the abscess could be achieved after continuing the proper antibiotic therapy.

Our surgical site infection rate percentage at our hospital is $0.1-0.4 \%$ which comes with the acceptable international rate [14].

\section{Abbreviations}

ESBL: Extended spectrum beta lactamase

MDR: Multidrug resistance

ER: Emergency room

HPF: High power field

SH: Stapled hemorrhoidopexy.

\section{Conflicts of Interest}

The authors declare that there are no conflicts of interest regarding the publication of this paper.

\section{Acknowledgments}

Authors are grateful to Laboratory Department of IMC Hospital, Microbiology team, and Dr. Ibrahim Mansoor and Dr. Ebtihal Soliman and Mr. Mohamed Alhaj for their help.

\section{References}

[1] J. M. Mccloud, J. S. Jameson, and A. N. D. Scott, "Lifethreatening sepsis following treatment for haemorrhoids: A systematic review," Colorectal Disease, vol. 8, no. 9, pp. 748-755, 2006.

[2] R. G. Molloy and D. Kingsmore, "Life threatening pelvic sepsis after stapled haemorrhoidectomy," Lancet, vol. 355, no. 9206, p. 810, 2000.

[3] V. Ripetti, M. Caricato, and A. Arullani, "Rectal perforation, retropneumoperitoneum, and pneumomediastinum after stapling procedure for prolapsed hemorrhoids: Report of a case and subsequent considerations," Diseases of the Colon and Rectum, vol. 45, no. 2, pp. 268-270, 2002.

[4] P. Roos, "Haemorrhoid surgery revised," Lancet, vol. 355, no. 9215, article 1648, 2000.

[5] G. D. Giebel, "Comment on Ch Bonner et al: Fournier gangrene as a rare complication after stapler haemorrhoidectomy. Case report and review of the literature," Chirurg, vol. 73, article 288, 2002.

[6] J. M. McCloud, H. Doucas, A. D. N. Scott, and J. S. Jameson, "Delayed presentation of life-threatening perineal sepsis following stapled haemorrhoidectomy: A case report," Annals of the Royal College of Surgeons of England, vol. 89, no. 3, pp. 301-302, 2007.

[7] A. Maw, R. Concepcion, K.-W. Eu et al., "Prospective randomized study of bacteraemia in diathermy and stapled haemorrhoidectomy," British Journal of Surgery, vol. 90, no. 2, pp. 222226, 2003.

[8] L. Wiese, X. Nielsen, K. Andresen, A. Kjaer, and K. David, "16s rDNA sequencing revealed Citrobacter freundii as the cause of liver abcess after banding of rectal haemorrhoids," Journal of Infection, vol. 50, no. 2, pp. 163-164, 2005.

[9] G. D. A. Santos, C. P. Coutinho, M. M. M. M. D. E. Meyer, D. V. Sampaio, and G. M. G. da Cruz, "Surgical complications in 2,840 cases of hemorrhoidectomy by Milligan-Morgan, Ferguson and combined techniques," Journal of Coloproctology, vol. 32, no. 3, pp. 271-290, 2012.

[10] P. Giordano, G. Gravante, R. Sorge, L. Ovens, and P. Nastro, "Long-term outcomes of Stapled Hemorrhoidopexy vs conventional Hemorrhoidectomy: A meta-analysis of randomizedcontrolled trials," Archives of Surgery, vol. 144, no. 3, pp. 266-272, 2009.

[11] R. Ben-Ami, J. Rodríguez-Baño, H. Arslan et al., "A multinational survey of risk factors for infection with extendedspectrum $\beta$-lactamase-producing enterobacteriaceae in nonhospitalized patients," Clinical Infectious Diseases, vol. 49, no. 5, pp. 682-690, 2009.

[12] M. Melzer and I. Petersen, "Mortality following bacteraemic infection caused by extended spectrum beta-lactamase (ESBL) producing E. coli compared to non-ESBL producing E. coli," Journal of Infection, vol. 55, no. 3, pp. 254-259, 2007.

[13] M. Tumbarello, T. Spanu, M. Sanguinetti et al., "Blood stream infections caused by extended-spectrum- $\beta$-lactamaseproducing Klebsiella pneumoniae: risk factors, molecular epidemiology, and clinical outcome," Antimicrobial Agents and Chemotherapy, vol. 50, no. 2, pp. 495-504, 2006.

[14] S. S. Lewis, R. W. Moehring, L. F. Chen, D. J. Sexton, and D. J. Anderson, "Assessing the relative burden of hospital-acquired infections in a network of community hospitals," Infection Control and Hospital Epidemiology, vol. 34, no. 11, pp. 1229-1230, 2013. 


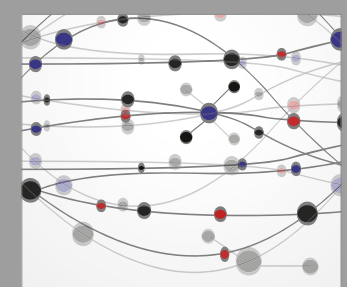

The Scientific World Journal
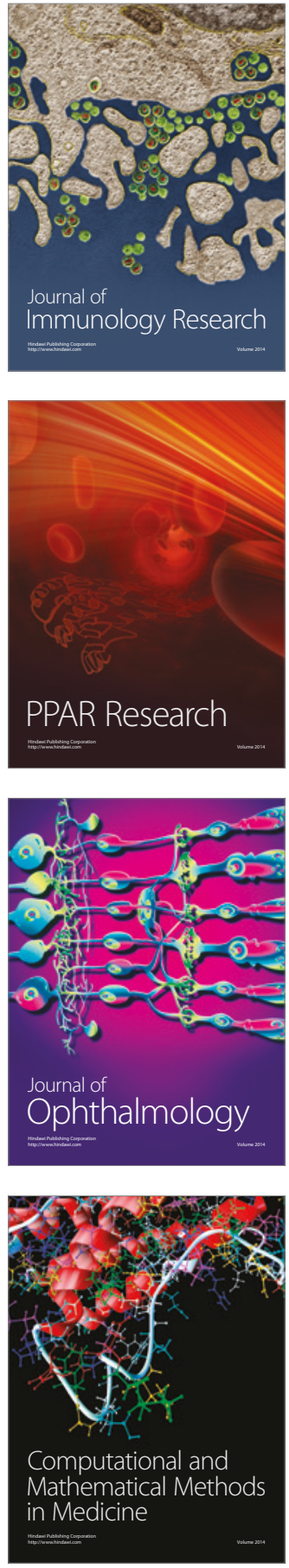

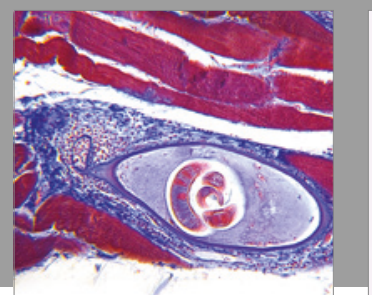

Gastroenterology Research and Practice
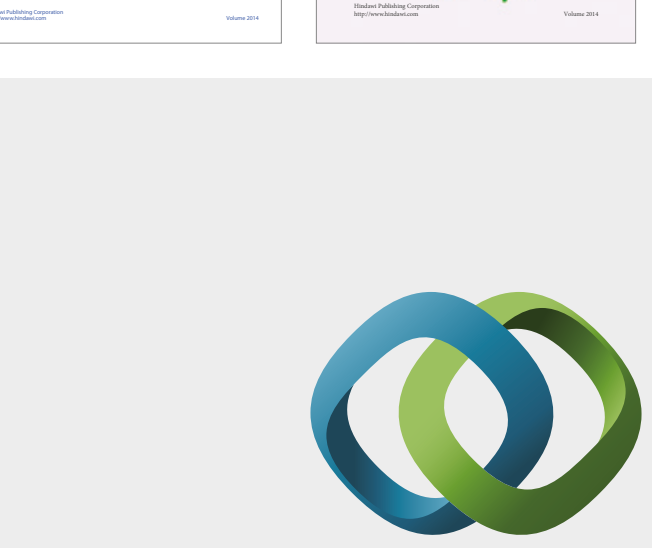

\section{Hindawi}

Submit your manuscripts at

https://www.hindawi.com
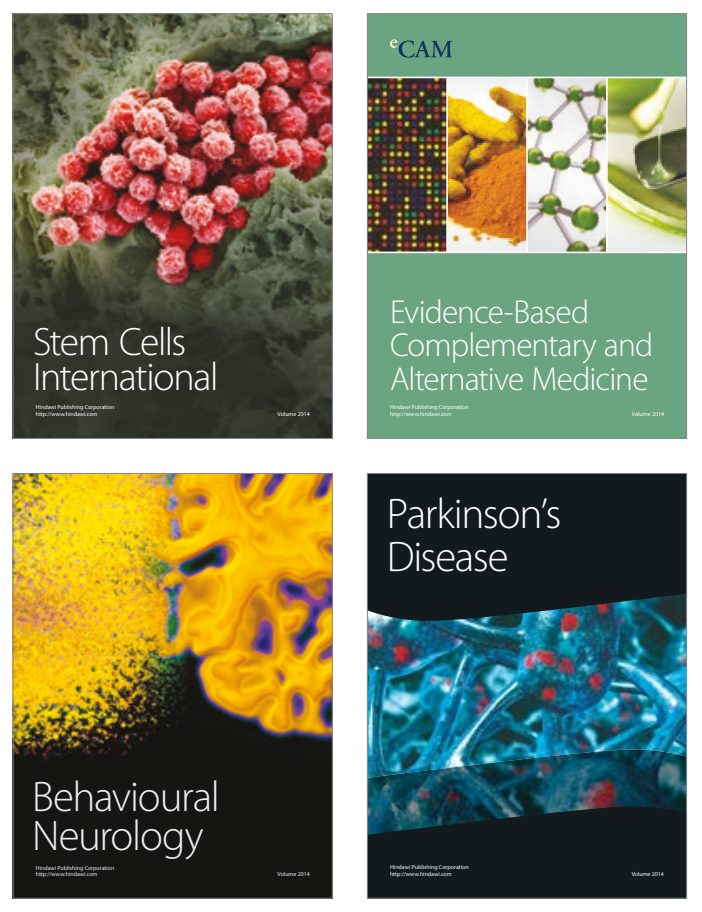
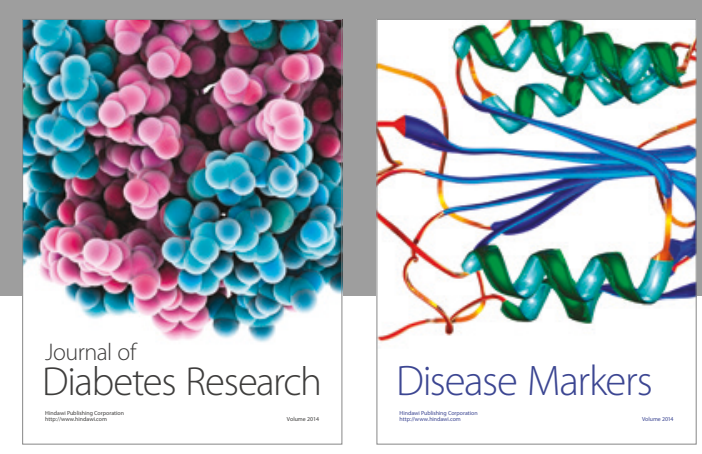

Disease Markers
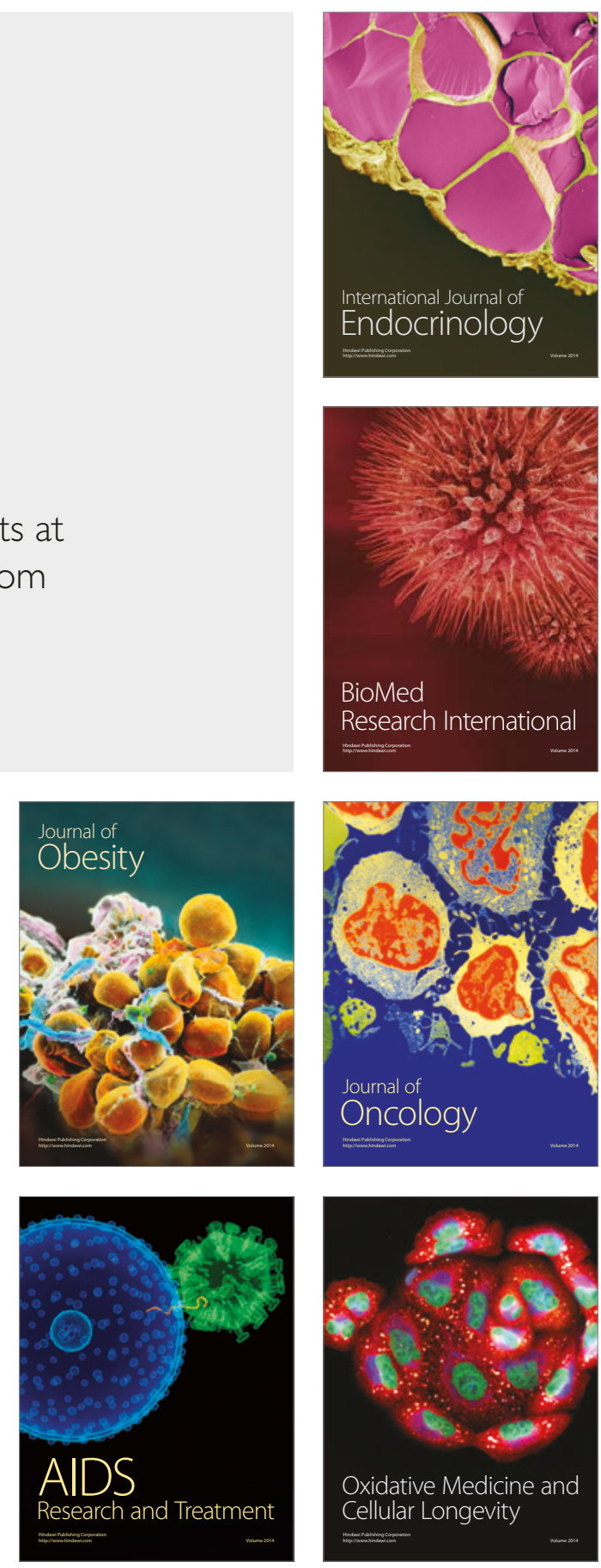